Nikolas Eisentraut

\title{
Die Digitalisierung von Forschung und Lehre - auf dem Weg in eine „öffentliche“ Rechtswissenschaft?
}

Übersicht

I. Der Stand der Digitalisierung von Forschung und Lehre: Eine kritische Bestandsaufnahme

1. Lehre

2. Forschung

II. Die Digitalisierung als Weg in eine „öffentliche“ Rechtswissenschaft?

1. Was ist öffentliche Wissenschaft?

a) Open Access

b) Open Educational Resources

2. Initiativen zur Förderung öffentlicher Wissenschaft

3. Rezeption in der Rechtswissenschaft

a) Offene Lehrmaterialien in der Rechtswissenschaft

b) Offene Forschung in der Rechtswissenschaft

c) Vorbehalte

aa) Fachkultur und Reputation

bb) Ökonomische Erwägungen

III. Lösungsansätze

1. Zwang durch Open-Access-Zweitverwertungspflichten

a) Zuständigkeit der Landesgesetzgeber

b) Vereinbarkeit mit Art 5 Abs. 3 S. 1 GG

aa) Schutzbereich

bb) Eingriff

cc) Rechtfertigung

(1) Art. 5 Abs. 3 GG in seiner objektiv-rechtlichen Dimension

(2) Art. 5 Abs. 3 GG als Grundrecht anderer

Wissenschaftler ${ }^{*}$ innen

(3) Informationsfreiheit

(4) Staatliche Finanzierung

(5) Abwägung

c) Vereinbarkeit mit Art. 14 Abs. 1 und 12 Abs. 1 GG

d) Vereinbarkeit mit der Urheberrechtsrichtlinie

2. Förderung

* Der Beitrag beruht auf einem Vortrag, den der Verfasser auf der 60. Assistententagung 2020 in Trier gehalten hat; er erscheint in einer gekürzten Fassung auch im Tagungsband. Soweit ein OpenAccess-Zugang besteht, werden in den Fußnoten Links zu den Beiträgen angegeben. Diese wurden zuletzt am 9.6.2020 auf ihre Gültigkeit überprüft.

1 Stifterverband/Heinz Nixdorf Stiftung, Hochschulbarometer 2019, S. 22, abrufbar unter https://www.stifterverband.org/medien/ hochschul-barometer-2019; die Digitalisierung berührt auch das Öffentliche Recht auf einer Vielzahl von Ebenen, so insbesondere unter dem Aspekt des E-Government, dazu zuletzt Siegel, EGovernment und das Verwaltungsverfahrensgesetz, DVBl 2020, S. 552; Guckelberger, Öffentliche Verwaltung im Zeitalter der Digitalisierung, 2019; Seckelmann, Digitalisierte Verwaltung, Ver-
3. Freiwilligkeit: $\$_{38}$ Abs. 4 UrhG

IV. Fazit

Mit den Hochschulen gerät auch die Rechtswissenschaft in Zugzwang, sich verstärkt in digitalen Räumen zu bewegen. Mit der Digitalisierung eng verknüpft ist die Forderung nach einer weiterreichenden Öffnung der (Rechts-)Wissenschaft. Neben Initiativen der Europäischen Kommission und des Bundes haben die Länder im Verbund mit ihren Hochschulen und Forschungsorganisationen weitreichende Strategien zur Öffnung der Wissenschaft aufgesetzt. Indes scheint die Entwicklung in der Rechtswissenschaft nur sehr zögerlich anzukommen. Der Beitrag spürt der Digitalisierung von rechtswissenschaftlicher Forschung und Lehre nach und sucht nach den Gründen für die geringe Verbreitung offener Publikationen. Zugleich setzt er sich auch mit den Grenzen einer zwangsweisen Öffnung rechtswissenschaftlicher Forschung und Lehre auseinander.

\section{Der Stand der Digitalisierung von Forschung und Lehre: Eine kritische Bestandsaufnahme}

Die Digitalisierung als Gegenstand der Forschung ist ein Großthema der letzten Jahre. ${ }^{1}$ Die Hochschulen reagieren und richten immer mehr Lehrstühle mit dem Schwerpunkt Digitalisierung ein: drei von zehn im Jahr 2018 neuberufene Professoren beschäftigen sich mit Themen der Digitalisierung. ${ }^{2}$

Die Hochschulen selbst haben indes Nachholbedarf was ihre eigene Digitalisierung angeht. Dies betrifft zunächst die Digitalisierung als Lehrinhalt. ${ }^{3}$ Aber auch für die umfassende Digitalisierung der Infrastrukturen der Hochschulen braucht es nach Auffassung der Hoch-

netztes E-Government, 2. Aufl. 2019, in Hinblick auf das Datenschutzrecht, s. nur Schmidt-Jorzig, IT-Revolution und Datenschutz, DÖV 2018, S. 10 und auch in Hinblick auf das Verfassungsrecht, s. nur die Beiträge aus jüngerer Zeit von Golla, In Würde vor Ampel und Algorithmus - Verfassungsrecht im technologischen Wandel, DÖV 2019, S. 673; Härtel, Digitalisierung im Lichte des Verfassungsrechts - Algorithmen, Predictive Policing, autonomes Fahren, LKV 2019, S. 49 und Schliesky, Digitalisierung - Herausforderung für den demokratischen Verfassungsstaat, NVwZ 2019, S. 693.

2 Stifterverband/Heinz Nixdorf Stiftung (Fn. 1), S. 23.

$3 \mathrm{Zu}$ den neuen digitalen Ausbildungsinhalten Zwickel, Jurastudium 4.0.? - Die Digitalisierung des juristischen Lehrens und Lernens, JA 2018, S. 881 (881 ff.). 
schulleitungen umfassender Investitionen. ${ }^{4}$ Wo bereits digitale Infrastrukturen für Forschung und Lehre bestehen, werden sie bisher zudem kaum genutzt.

\section{Lehre}

In Hinblick auf die Lehre halten die Hochschulen zwar digitale Lernplattformen vor. ${ }^{5}$ Auf diesen Lernplattformen werden jedoch meist nur Basis-Lehrmaterialien angeboten wie Vorlesungsskripte, Präsentationen, Forschungsliteratur im Rahmen des $₫$ 6oa UrhG (etwa der ein oder andere zur Vertiefung gedachte Aufsatz oder ein Lehrbuchauszug) und Falllösungen. Meist sind die digitalen Lernumgebungen zudem verschlossen: Nur die Kursteilnehmer*innen erhalten Zugang zu den Materialien. Darüberhinausgehende E-Learning-Angebote, die Formen klassischer Lehre ersetzen könnten, finden sich bisher nur in Ansätzen. ${ }^{6}$

Umfassenderes und wissenschaftlich aufbereitetes Lehrmaterial wie Lehr- und Fallbücher, aber auch Aufsätze in Ausbildungszeitschriften werden bisher hingegen weitgehend "privatisiert" und unterliegen kommerziellen Interessen. Kostenpflichtige Lehrbücher werden zur Lektüre für die Klausur- und Examensvorbereitung anempfohlen und genießen den Status seriöser Lehrmaterialien, während frei im Internet zirkulierenden Skripten wenig Vertrauen in die Qualität entgegengebracht wird.?

Diese bisher überwiegend als Printfassung verfügbaren kommerziellen Lehrmaterialien treten nur langsam den Weg ins digitale Zeitalter an: Lehrbücher erscheinen zuletzt vermehrt auch als digitale Ausgaben in den For-

4 Stifterverband/Heinz Nixdorf Stiftung (Fn. 1), S. 17 und spezifisch für die Lehre S. 19; Investitionsbedarfe wird auch die BlockchainTechnologie auslösen, dazu Schürmeier, Blockchain-Anwendungen in der Hochschule, DVP 2019, S. 409 sowie Camilleri/ Werner/Hoffknecht/Sorge, Blockchain in der Hochschulbildung, 2019, abrufbar unter https://www.stifterverband.org/blockchainin-der-hochschulbildung.

5 Näher zum Stand der Implementierung von CMS- und LMSSystemen Expertenkommission Forschung und Innovation (Hrsg.), Digitalisierung der Hochschulen, 2019, S. 47, Studie abrufbar unter https://www.e-fi.de/fileadmin/Innovationsstudien_2019/ StuDIS_14_2019.pdf; Zu den datenschutzrechtlichen Fragen bei E-Learning-Plattformen Botta, Datenschutz bei E-LearningPlattformen, 2020 passim.

6 Zwickel (Fn. 3), S. 881 (884); Reiner, Juristische Didaktik und E-Lernen: Theoretische Konzeption und Anwendungsbeispiele, JurPC Web-Dok 160/2007, Abs. 1, abrufbar unter https://www. jurpc.de/jurpc/show?id=20070160; ein positives Bild zeichnet Meyer, Urheberrecht und Wissensgesellschaft - innovative und zeitgemäße Lehr- und Lernformen im Spiegel des Urheberrechtsschutzes, WissR 2018, S. 224 (224) ; Die Corona-Krise hat der Digitalisierung der Lehre einen Schub gegeben, dessen langfristige Wirkung sich aber erst zeigen wird, hierzu Eisentraut, Corona als Chance für die Digitalisierung rechtswissenschaftlicher Forschung und Lehre, JuWissBlog Nr. 30/2020 v. 20.3.2020, abrufbar unter https://www.juwiss.de/30-2020/. maten epub und $p d f$ und stehen bei Kauf der Printversion zum Download zur Verfügung. Die Verlage schaffen zudem Plattformen, auf denen Literatur eingesehen und heruntergeladen werden kann, etwa Ausbildungszeitschriften, soweit sie denn von der jeweiligen Universitätsbibliothek auch im digitalen Format lizenziert wurden. ${ }^{8}$

\section{Forschung}

Im Bereich der Forschung bietet sich ein ganz ähnliches Bild: Die Hochschulen halten zwar Publikationsserver vor, die einen einfachen und kostenlosen Upload von Forschungsergebnissen ermöglichen würden. ${ }^{9}$ Weite Teile juristischer Forschungsergebnisse werden indes "privatisiert" und bleiben dabei im Analogen verhaftet. Dies zeigt sich schon an den Scan- und Kopierarbeiten, die die eigene Forschung regelmäßig erforderlich macht. Wo digitale Verlagsangebote vorhanden sind, hängt der Zugang von der Lizenzierung durch die Universitätsbibliotheken ab. Ansonsten verschwinden digitale Forschungsergebnisse hinter Bezahlschranken.

\section{Die Digitalisierung als Weg in eine „öffentliche“ Rechtswissenschaft?}

Handelt es sich bei der Digitalisierung von Forschung und Lehre also eigentlich nur um alten Wein in neuen Schläuchen? Wird der "beschwerliche" Weg der analogen Literaturrecherche im digitalen Raum nunmehr durch Bezahlschranken versperrt?

$7 \quad \mathrm{Zu}$ diesem Ergebnis kam eine gemeinsame Studie von Hanser Fachbuch und Studierenden der Universität Hannover, deren Ergebnisse hier dargestellt werden: https://www.boersenblatt. net/2019-02-06-artikel-gemeinsame_studie_von_hanser_fachbuch_und_medienmanagement-studenten.1593210.html; zu möglichen künftigen Qualitätskriterien für offen verfügbare Bildungsmaterialien Barthelmeß, E-Learning - bejubelt und verteufelt, 2015, S. 73.

8 Ein Grund für die zögerliche Digitalisierung mag die Wahrung der Urheberrechte im digitalen Raum darstellen, vgl. Gercke, in: Spindler/Schuster, Recht der elektronischen Medien, 4. Aufl. 2019, Vorbemerkung zu $\$ \$ 106$ ff. Rn. 5; die Gefahr des Internets als „Plattform für die illegitime Aneignung fremder Schöpfungen“ adressiert auch Bäuerle, Open Access zu hochschulischen Forschungsergebnissen?, in: Britz (Hrsg.), Forschung in Freiheit und Risiko, 2012, S. 1 (3); s. auch Braun, Die Rückkehr der Autoren, Blätter für deutsche und internationale Politik 1/2011, S. 101 (101) sowie Steinhauer, Die Nutzung einer „Schattenbibliothek“ im Licht des Urheberrechts, 2016, abrufbar unter https:// ub-deposit.fernuni-hagen.de/receive/mir_mods_00000825\#.

9 Eine Übersicht über alle verfügbaren Repositorien und ihre jeweilige institutionelle Anbindung findet sich unter https://dini. de/dienste-projekte/dini-zertifikat/liste-der-repositorien/; zur Implementation einer digitalen Infrastruktur durch die Hochschulen Expertenkommission Forschung und Innovation (Hrsg.) (Fn. 5), S. 43. 
1. Was ist öffentliche Wissenschaft?

Eine - in der Rechtswissenschaft bisher nicht ausreichend gewürdigte - Bewegung drängt auf eine alternative Nutzung der Möglichkeiten der Digitalisierung. Stellen wir uns eine Welt vor, in der jeder Aufsatz, jede Monographie und jedes Lehrbuch digital frei zugänglich verfügbar ist. Verlinkte Fußnoten und Literaturlisten würden so zu interaktiven Landkarten, die mit einem Klick den Eintritt in ein ganzes Wissenschaftsuniversum ermöglichten. Lehrbücher stünden zur Nachnutzung offen, sodass kursbegleitende Materialien ohne Konflikte mit dem Urheberrecht den Studierenden bereitgestellt und um relevante Quellen ergänzt werden könnten.

Als Oberbegriff einer solchermaßen verstandenen Wissenschaft hat sich der Terminus „Open Science“ oder auch „Öffentliche (auch: offene) Wissenschaft" etabliert. ${ }^{10}$

Die Idee einer öffentlichen Wissenschaft betont die Bedeutung von Wissen als kulturellem Gemeingut und ist damit anschlussfähig an die Debatte um Gemeingüter. ${ }^{11}$ Das Konzept gerät dadurch in eine Spannungslage zum Schutz wissenschaftlicher Werke als geistiges Eigentum durch das Urheberrecht. ${ }^{12}$ Das UrhG reagiert auf diese Spannungslage mehrfach und enthält Regelungen, die einen Ausgleich zwischen urheberrechtlichem Eigentum und dem Interesse der Allgemeinheit an der Nutzung wissenschaftlicher Leistungen schaffen sollen, s. das Zitatrecht in $\$ 51$ UrhG, $\$ 60 a$ UrhG speziell für die Hochschullehre sowie $₫ 60 c$ und $₫$ 6od UrhG für die
Nutzung urheberrechtlich geschützter Inhalte im Rahmen der Forschung. ${ }^{13}$ Konzepte öffentlicher Wissenschaft streben jedoch eine Öffnung über die Schrankenregelungen des UrhG hinaus an.

\section{a) Open Access}

Eine zentrale Rolle kommt in den Rechtswissenschaften der Idee einer freien Verfügbarkeit von Forschungsliteratur zu, die unter dem Schlagwort „Open Access“ firmiert. Open Access zeichnet aus, dass mit der Veröffentlichung allen die Erlaubnis erteilt wird, das OpenAccess-veröffentlichte Dokument zu lesen, herunterzuladen, zu speichern, es zu verlinken, zu drucken und damit entgeltfrei zu nutzen. ${ }^{14}$ Unterschieden wird zwischen dem sog. goldenen und dem grünen Weg: Während beim goldenen Weg bereits die Erstveröffentlichung Open Access erfolgt, wird beim grünen Weg häufig nach einer klassischen Verlagsveröffentlichung auch ein Open-Access-Zugang eröffnet. ${ }^{15}$ $\$ 38$ Abs. 4 UrhG räumt hierfür ein Recht zur nichtkommerziellen Zweitveröffentlichung ein.

Open Access verfolgt die Idee neuer Finanzierungsformen für wissenschaftliche Literatur. Während bisher eine Nutzerfinanzierung vorherrschend ist, soll die Leserschaft künftig von Zugriffskosten befreit werden. ${ }^{16}$ Open Access ist damit auch eine Reaktion auf immer weiter ansteigende Abonnement-Gebühren für juristische Zeitschriften, die die Bibliotheksetats zu erschöpfen drohen und soll die Mehrfachsubventionierung hochschulischer Forschung beenden. ${ }^{17}$ Open Access fordert
10 S. auch zu den weitergehenden Gehalten des Begriffs Hamann/ Goller/Havemann u.a., Handbuch Open Science, erschienen auf Wikibooks, abrufbar unter https://de.wikibooks.org/wiki/Handbuch_Open_Science.

11 Bäuerle (Fn. 8), S. 1 (3); Dobusch/Heimstädt, Erst Offenheit gibt digitaler Bildung Richtung, Forum Wissenschaft 4/16, S. 21 (21), abrufbar unter http://www.dobusch.net/pub/uni/DobuschHeimstaedt(2016)Forum_Wissenschaft-BDWI-OER.pdf; Baer, Braucht das Grundgesetz ein Update?, Blätter für deutsche und internationale Politik 1/2011, S. 92 (92); Allgemein zum Thema Gemeingüter näher Helfrich, Wem gehört die Welt? Zur Wiederentdeckung der Gemeingüter, 2009, abrufbar unter https://www. boell.de/sites/default/files/assets/boell.de/images/download_de/ Netzausgabe_Wem_gehoert_die_Welt.pdf; s. auch Hofmann (Hrsg.), Wissen und Eigentum, 2006, abrufbar unter https://www. bpb.de/shop/buecher/schriftenreihe/36112/wissen-und-eigentum.

12 S. nur Schulze, in: Dreier/Schulze, Urheberrechtsgesetz, 6. Aufl. 2018, § 2 Rn. 41 sowie 93 ff.; Schmidt, Open Access, 2016, S. 160 ff.

13 Die Auswirkungen dieser Vorschriften auf die Hochschullehre untersucht Meyer (Fn. 6), S. 224 passim.

14 Zum Begriff des Open Access Eisentraut, Open Access in der Rechtswissenschaft, RBD 2018, 87 (87), abrufbar unter http:// dx.doi.org/10.17169/refubium-26494; Haug, Open Access in Baden-Württemberg: Rechtswidriger Zweitveröffentlichungszwang zwischen Urheber- und Hochschulrecht, OdW 2019, S. 89 (89), abrufbar unter http://ordnungderwissenschaft.de/wpcontent/uploads/2019/11/12_02_2019_haug_open_access_odw. pdf; zur Historie näher Bäuerle (Fn. 8), S. 1 (1 f.); näher mit den möglichen Nutzungsrechten setzt sich auseinander Peukert, Ein wissenschaftliches Kommunikationssystem ohne Verlage - zur rechtlichen Implementierung von Open Access als Goldstandard wissenschaftlichen Publizierens, Goethe Universität Frankfurt, Fachbereich Rechtswissenschaft, Arbeitspapier Nr. 6/2013, S. 10 ff., abrufbar unter http://publikationen.ub.uni-frankfurt.de/ frontdoor/index/index/docId/29488.

15 Ulmer/Eilfort/Obergfell, Verlagsrecht, 2013, Kapitel L Rn. 15; Haug (Fn. 14), S. 89 (89); Götting/Lauber-Rönsberg, Open Access und Urheberrecht, OdW 2015, S. 137 (137 f.), abrufbar unter http://ordnungderwissenschaft.de/wp-content/uploads/2020/03/19_goetting_lauber-roensberg_open-access_und_ urheberrecht_2015.pdf.

16 Krausnick, Offene Wissenschaft? - Öffentlich-rechtliche Aspekte der Diskussion um Open Access und Open Data, in: Festschrift Friedhelm Hufen, 2015, S. 367 (369).

17 Bäuerle (Fn. 8), S. 1 (5). 
damit zugleich die hergebrachten Geschäftsmodelle der etablierten Verlage heraus. ${ }^{18}$

\section{b) Open Educational Resources}

Eine weitere Teilmenge öffentlicher Wissenschaft ${ }^{19}$ stellen sog. „Open Educational Resources ${ }^{\alpha_{20}}$ (OER) dar, die auf eine Öffnung der Lehre im Sinne einer freien Verfügbarkeit von Lehr- und Lernmaterialien abzielen. ${ }^{21}$ Für die Hochschullehre werden OER große Potentiale zugemessen. ${ }^{22}$ Mit ihnen lassen sich einerseits die Potentiale digitaler Bildung entfalten, andererseits ermöglichen OER eine neue Form der Zugänglichkeit von Bildungsangeboten: Sie erlauben die kollaborative Er- und Bearbeitung von Lehr- und Lernmaterialien durch Lehrende und Studierende gemeinsam und führen zu Qualitätsund Effizienzgewinnen durch eine sinnvolle Nachnutzung bereits erstellter Materialien. ${ }^{23}$ Dadurch kann die auch an Hochschulen oftmals nur knapp bemessene Zeit in die Verbesserung von Lern- und Lehrmaterialien investiert werden, anstatt in die Ausarbeitung immer wieder derselben grundlegenden Materialien. Die freie Nachnutzbarkeit ermöglicht es zudem, dass die Materialien in eine Vielzahl unterschiedlicher Lernangebote eingebunden werden können: Vom klassischen Lernen mit

18 Bäuerle (Fn. 8), S. 1 (8); die Möglichkeit nachhaltiger OpenAccess-Geschäftsmodelle betont Rux, Open Access im rechtswissenschaftlichen Verlag, in: Hamann/Hürlimann, Open Access in der Rechtswissenschaft, Sonderheft der Zeitschrift „Rechtswissenschaft“, 2019, abrufbar unter https://doi. org/10.5771/9783748903659; jedenfalls zugangskontrollierte Datenbanken werden in einer Open-Access-Zukunft keine Rolle mehr spielen, vgl. Peukert (Fn. 14), S. 6.

19 Im Sinne des verfassungsrechtlichen Wissenschaftsbegriffs (vgl. dazu BVerfG, Urt. v. 29.5.1973, Az.: 1 BvR 424/71 und 325/72 = BVerfGE 35, 79, abrufbar unter http://www.servat.unibe.ch/dfr/ bv035079.html Rn. 129), sollten auch Open Access und Open Educational Resources als Ausprägungen ein und desselben Gegenstandes verstanden werden; zum Verhältnis von Open Educational Resources und Open Access Deimann/Neumann/MuußMerholz, Whitepaper Open Educational Resources (OER) an Hochschulen in Deutschland - Bestandsaufnahme und Potentiale 2015, S. 32, abrufbar unter https://open-educational-resources. de/wp-content/uploads/Whitepaper-OER-Hochschule-2015.pdf.

20 Eisentraut, Open Educational Resources in der Rechtswissenschaft, RBD 2018, S. 93, abrufbar unter http://dx.doi. org/10.17169/refubium-26489; Deimann/Neumann/Muuß-Merholz (Fn. 19), S. 10.

21 Näher zu den Definitionsansätzen Deimann/Neumann/MuußMerholz (Fn. 19), S. 10.

22 Eckhoff, OER in der Hochschulbildung, OERinfo - Informationsstelle OER, abrufbar unter https://open-educational-resources.de/ dossierseite/?praxis=allgemein\&bereich $=$ hochschule.

23 Wikimedia Deutschland e.V. (Hrsg.), Praxisrahmen für Open Educational Resources (OER) in Deutschland, 2016, S. 7, abrufbar unter http://mapping-oer.de/wp-content/uploads/2016/02/ Praxisrahmen-fu\%CC\%88r-OER-in-Deutschland_Online.pdf.

24 Als weitere Teilmengen offener Wissenschaft kommt den Themen Open Data und Wissenschaftskommunikation für die Rechts- einem gedruckten Lehrbuch, über das Lernen mit einer intelligent verlinkten Online-Ressource, bis hin zur Auseinandersetzung mit dem Lernstoff in interaktiven Onlinekursen oder über eine App. ${ }^{24}$

\section{Initiativen zur Förderung öffentlicher Wissenschaft}

Gerade das Thema Open Access ist sowohl in der Politik als auch an den Universitäten dabei, Mainstream zu werden. Ausgehend von der Berliner Erklärung über den offenen Zugang $\mathrm{zu}$ wissenschaftlichem Wissen v. 22.10.2003, die mittlerweile von 654 Institutionen unterzeichnet wurde, ${ }^{25}$ befürwortet heute eine große Mehrheit der Hochschulleitungen eine Öffnung ihrer Forschung i.S.v. Open Access: 93,8 \% der Hochschulleitungen stimmen zu, dass sie Open Access als Standard künftig fördern werden. ${ }^{26}$ Ausdruck findet die Öffnung zudem in den Open-Access-Strategien der Hochschulen. ${ }^{27}$ Auch die großen Forschungsorganisationen haben sich zu Open Access bekannt. ${ }^{28}$

Und auch in der Politik ist das Thema Open Access angekommen. Eine Öffnung der Forschung wird von Seiten der Politik nicht nur stärker gefördert, sondern zunehmend auch gefordert. ${ }^{29}$ Mehrere Bundesländer haben eigene Open-Access-Strategien verabschiedet. ${ }^{30} \mathrm{Die}$

wissenschaft Bedeutung zu, s. zu Open Data näher Krausnick (Fn. 16), S. 367 (368); Eisentraut/Hamann, Handbuchbeitrag Open Science und Rechtswissenschaft, Abschnitt Open Data als Praxis der Rechtswissenschaft, in: Handbuch Open Science, erschienen auf Wikibooks, abrufbar unter https://de.wikibooks. org/wiki/Handbuch_Open_Science/_Rechtswissenschaft und zur Wissenschaftskommunikation Weitze/Heckl, Wissenschaftskommunikation, 2016 - passim. Zur zunehmenden Bedeutung von Open Data s. die „Sorbonne-Erklärung für offene Forschungsdaten“, abrufbar unter https://www.german-u15.de/presse/ressourcen-2020/20200130_Sorbonne-Declaration-on-Research-DataRights.pdf; für die Bedeutung der Wissenschaftskommunikation s. das Grundsatzpapier des Bundesministeriums für Bildung und Forschung zur Wissenschaftskommunikation, November 2019,

S. 3, abrufbar unter https://www.bmbf.de/upload_filestore/pub/ Grundsatzpapier_zur_Wissenschaftskommunikation.pdf.

25 Die Liste der Institutionen ist abrufbar unter https://openaccess. mpg.de/3883/Signatories.

26 Stifterverband/Heinz Nixdorf Stiftung (Fn. 1), S. 26.

27 S. nur beispielhaft die Open-Access-Strategie 2018 - 2020 der Freien Universität Berlin, abrufbar unter http://dx.doi. org/10.17169/FUDOCS_document_000000028882; zu sog. „Open-Access-Mandates“ der Hochschulen Götting/LauberRönsberg (Fn. 15), S. 137 (138 f.).

28 Die Positionen finden sich verlinkt unter https://www.fu-berlin. de/sites/open_access/weiteres/oa_positionen/index.html.

29 Zuletzt etwa Karliczek, Rede der Bundesministerin für Bildung und Forschung anlässlich des Forschungsgipfels 2018 in Berlin, abrufbar unter https://anja-karliczek.de/rede-der-bundesministerin-fuer-bildung-und-forschung-anja-karliczek-anlaesslich-desforschungsgipfels-2018-in-berlin/.

30 Die Strategien sind verlinkt unter https://www.fu-berlin.de/sites/ open_access/weiteres/oa_positionen/index.html. 
Zielvorgabe des Landes Berlin für den Umfang an OpenAccess publizierten Zeitschriftenartikeln liegt in diesem Jahr bei $60 \% .^{31}$ In Baden-Württemberg werden die Hochschulen sogar im Hochschulgesetz dazu angehalten, ihr wissenschaftliches Personal zu Open-AccessZweitveröffentlichungen zu verpflichten. Auch das Bundesministerium für Bildung und Forschung hat eine Open-Access-Strategie veröffentlicht. ${ }^{32}$

Darüber hinaus richtet sich auch die europäische Wissenschaftslandschaft auf eine Öffnung ein. ${ }^{33}$ In der Forschungsförderung Horizont 2020 ist die Open-Access-Publikation der geförderten Projektergebnisse verpflichtend. ${ }^{34}$

Schließlich entstehen auch auf gesellschaftlicher Ebene Förderprogramme für Wissenschaftler*innen, die eine Öffnung ihrer Wissenschaft forcieren, etwa das von Wikimedia, dem Stifterverband und der VolkswagenStiftung getragene Open Science Fellowship, dessen Fellow der Autor dieses Beitrags im letzten Jahr sein durfte. ${ }^{35}$

Im Bereich OER entsteht zurzeit eine weitreichende digitale Infrastruktur für das Angebot offener Bildungsmaterialien. Aktuell existieren sieben hochschulübergreifende Plattformen, die das Potential haben, zu digitalen Hochschulen zu werden. Herausgehoben seien an dieser Stelle die Plattform „Hamburg Open Online University“, die sich als offene Plattform für hochschulübergreifende Angebote und Lernprojekte der Hamburger Hochschulen begreift ${ }^{36}$ und die VHB, die virtuelle Hochschule Bayern, auf der eingeschriebenen Studierenden kostenfreie, digitale Ergänzungskurse angeboten werden und die sich kürzlich auch für die Allgemeinheit geöffnet hat. ${ }^{37}$ Auch die Europäische Kommission plant eine Plattform für digitale Hochschulbildung, eine Art „European Digital University“. ${ }^{38}$

\section{Rezeption in der Rechtswissenschaft}

Dass Wissenschaft und Öffentlichkeit zwei Seiten einer Medaille sind, ist eigentlich auch in der Rechtswissenschaft keine neue Idee: In der Grundrechtsdogmatik wird die „Öffentlichkeit von Wissenschaft“ gar als Funktionsbedingung für den wissenschaftlichen Diskurs qualifiziert, um Überprüfbarkeit und Kritisierbarkeit zu gewährleisten. ${ }^{39}$ Die Wissenschaftsfreiheit in Art. 5 Abs. 3 S. 1 GG wird insofern als Kommunikationsgrundrecht verstanden ${ }^{40}$ und Wissenschaft als Kommunikationszusammenhang. ${ }^{41}$

Dennoch greifen die Rechtswissenschaftler*innen bei den neuen Instrumenten der Digitalisierung nicht beherzt $\mathrm{zu}$, um ihre Forschung und Lehre nun endlich einer weiterreichenden Öffentlichkeit zuzuführen.

\section{a) Offene Lehrmaterialien in der Rechtswissenschaft}

Offene Lehrmaterialien sind bisher „nicht aus der ,idealistischen Wolke' in der Praxis der Hochschulen angekommen (...), wenn auch engagierte Einzelpersonen und bestimmte Institutionen daran arbeiten" ${ }^{\text {"42 }}$. Die digitalisierte Lehre fristet ein Nischendasein. ${ }^{43}$ Wirklich offene Lehrprojekte lassen sich bisher an einer Hand abzählen. Erste Leuchtturmprojekte zeigen indes die Potentia-
31 Senat von Berlin, Open-Access-Strategie für Berlin, Drucksache $17 / 2512$ v. 21.10.2015, S. 5, abrufbar unter http://dx.doi. org/10.17169/refubium-26319.

32 Open Access in Deutschland - Die Strategie des Bundesministeriums für Bildung und Forschung 2016, abrufbar unter https:// www.bmbf.de/upload_filestore/pub/Open_Access_in_Deutschland.pdf.

33 Die europäischen und internationalen Positionen zu Open Access finden sich verlinkt unter https://www.fu-berlin.de/sites/open_ access/weiteres/oa_positionen/index.html.

34 S. die Guidelines to the Rules on Open Access to Scientific Publications and Open Access to Research Data in Horizon 2020 v. 21.3.2017, abrufbar unter https://ec.europa.eu/research/participants/data/ref/h2020/grants_manual/hi/oa_pilot/h2020-hi-oapilot-guide_en.pdf.

35 Nähere Informationen zum Fellow Programm finden sich unter https://de.wikiversity.org/wiki/Wikiversity:Fellow-Programm_ Freies_Wissen.

36 Erreichbar unter https://www.hoou.de/.

37 S. https://www.vhb.org/open-vhb/; weitere Plattformen sind benannt in der Antwort der Bundesregierung auf eine kleine Anfrage zur Planung und Ausgestaltung einer europäischen Plattform für digitale Hochschulbildung, BT-Drs. 19/10106 v. 10.5.2019, S. 6, abrufbar unter https://dip21.bundestag.de/dip21/ btd/19/101/1910106.pdf.

38 Aktionsplan für digitale Bildung, Mitteilung der Europäischen
Kommission v. 17.1.2018, COM(2018) 22 final, abrufbar unter https://eur-lex.europa.eu/legal-content/DE/TXT/HTML/?uri=CE LEX:52018DC0022\&from=DE.

39 Bäuerle (Fn. 8), S. 1 (4); Gurlit, Transparenz in der Hochschulforschung, in: Festschrift Friedhelm Hufen, 2015, S. 343 (343); Ruffert, Grund und Grenzen der Wissenschaftsfreiheit, VVDStRL 65 (2006), 142 (184 ff.), abrufbar unter https://www.degruyter.com/downloadpdf/boo ks/9783110927832/9783110927832.146/9783110927832.146.pdf; Weingart, Die Wissenschaft der Öffentlichkeit, 2005, S. 9 ff.; auf das Wesen der Wissenschaft als „Gespräch, das über alle räumlichen und zeitlichen Grenzen hinweg geführt wird" weist bereits hin Kimminich, Das Veröffentlichungsrecht des Wissenschaftlers, Wissenschaftsrecht, Wissenschaftsverwaltung, Wissenschaftsförderung 1985, S. 116 (117).

40 Gärditz, in: Maunz/Dürig, GG, 88. EL August 2019, Art. 5 Abs. 3 Rn. 111 (Stand: 88. Lfg. August 2019).

41 S. nur Steinhauer, Das Recht auf Sichtbarkeit, 2010, S. 55, abrufbar unter https://www.infodata-edepot.de/volltext/aueintrag/10497.pdf.

42 Deimann/Neumann/Muuß-Merholz (Fn. 19), S. 15; s. auch Eisentraut (Fn. 20), S. 93.

43 Zwickel (Fn. 3), S. 881 (884); der zeitweise prekäre Zugang zu Ausbildungsliteratur während der Corona-Krise hat die Bedeutung offen zugänglicher Lehrmaterialien unterstrichen, vgl. Eisentraut (Fn. 6). 
le offener Lehre für die Rechtswissenschaft auf: Ein Projekt an der Bucerius Law School etwa hat offen lizenzierte Lehrvideos zu Grundfragen des Verwaltungsrechts produziert und offen lizenziert online gestellt. ${ }^{44}$ Über die Videos hinaus haben die Studierenden Wissenstests und teilweise auch Skripten erstellt, sodass eine medienübergreifende Lernumgebung entsteht.

Die Ludwig-Maximilians-Universität München bietet ein Portal für E-Learning an, das insbesondere mit online abrufbaren Podcasts von Vorlesungen heraussticht. ${ }^{45}$ Die WWU Münster bietet mit dem Modul „unirep PLUS“ digitale Lernressourcen einschließlich OnlineLektionen an, die jedoch nur für Studierende der WWU zugänglich sind. ${ }^{46}$ Beide Universitäten wurden dafür kürzlich mit dem Digital Award 2019 ausgezeichnet. ${ }^{47}$

Der Verfasser dieses Beitrags ist Herausgeber des ersten offen lizenzierten und damit zur Nachnutzung kostenlos zur Verfügung stehenden Lehrbuchs und eines das Lehrbuch begleitenden Fallrepetitoriums zum Verwaltungsrecht. Neben der Verlagsveröffentlichung im Print und zum kostenlosen digitalen Download ${ }^{48}$ sind beide Bücher auch online auf Wikibooks verfügbar, ${ }^{49}$ was es möglich macht, die von der Bucerius Law School erstellten Videos in den Lehrbuchcontent einzubinden. Solchen sog. MOOCS (Massive Open Online Courses) werden große Potentiale für die Hochschulbildung zugemessen..$^{\circ}$

44 Die Videos können hier abgerufen werden: https://www.youtube. com/playlist?list=PLPJgLCEwWmcNR6yf5VaN8AdrxAdGEe5jM; es steht nicht zu befürchten, dass Videoangebote die klassische Lehre im Hörsaal vollständig verdrängen werden, allein schon weil aufgezeichnete Vorlesungen eine Interaktion mit dem Dozierenden nicht erlauben; vielmehr erweitern solche Angebote die Möglichkeiten medienübergreifenden Lernens, vgl. Zwickel (Fn. 3), S. 881 (885).

45 Das Angebot findet sich unter https://www.jura.uni-muenchen. de/studium/e-learning/index.html; die Verknüpfung zur Virtuellen Hochschule Bayern weist jedoch noch keinerlei Kursangebote auf.

46 Abrufbar unter https://www.unirep-online.de/goto_unirep_ cat_591.html.

47 S. näher zur Digital Study 2019 https://lex-superior.com/digitalstudy/.

48 Das Lehrbuch „Verwaltungsrecht in der Klausur“ kann kostenlos über die Verlagshomepage von De Gruyter unter https://www. degruyter.com/view/title/565392?tab_body=toc-62810 und das Fallrepetitorium über die Verlagshomepage des Carl Grossmann Verlags unter http://www.carlgrossmann.com/?p=11914 heruntergeladen werden.

49 Abrufbar unter https://de.wikibooks.org/wiki/Verwaltungsrecht_ in_der_Klausur.

50 Zwickel (Fn. 3), S. 881 (884 f.); kritisch aber in Hinblick auf die oft restriktive Lizenzierung Dobusch/Heimstädt, (Fn. 11) S. 21 (24); s. b) Offene Forschung in der Rechtswissenschaft

Während das Thema Open Access immerhin in den rechtswissenschaftlichen Debatten angekommen ist, ${ }^{51}$ fehlt es auch hier noch einem nennenswerten Rückgriff auf diese Form der Publikation. Es gibt zwar OpenAccess-Zeitschriften; ${ }^{52}$ nur den wenigsten ist es jedoch bisher gelungen, ein den etablierten, im Subskriptionsmodell erscheinenden Zeitschriften vergleichbares Standing zu erlangen..$^{53}$ Erfolgreicher sind neue Formate wie Blogs und Podcasts, die von Beginn an auf eine weitreichende Öffentlichkeit im Digitalen gesetzt haben. Im öffentlichen Recht konnten sich etwa der JuWissBlog, ${ }^{54}$ der Verfassungsblog ${ }^{55}$ sowie der Völkerrechtsblog ${ }^{56}$ etablieren. Daneben existieren Preprintserver und universitäre Repositorien, die in der Rechtswissenschaft jedoch bisher weitgehend ein Schattendasein führen. ${ }^{57}$

\section{c) Vorbehalte}

Warum tut sich die Rechtswissenschaft so schwer mit dem Aufbruch ins neue digitale Zeitalter?

\section{aa) Fachkultur und Reputation}

Einen zentralen Faktor bildet die bisher fehlende Fachkultur: Open Science ist trotz aller politischen Bemühungen in der Rechtswissenschaft bisher ein Nischenthema; offene Veröffentlichungsformen sind nicht bereits zum Thema MOOCs auch einführend Botta (Fn. 5), S. 37 ff.

51 S. nur Hamann/Hürlimann, Open Access in der Rechtswissenschaft, Sonderheft der Zeitschrift „Rechtswissenschaft“, 2019, abrufbar unter https://doi.org/10.5771/9783748903659; Wildgans, Zuckerbrot oder Peitsche? - Ein Plädoyer für Open Access im juristischen Publikationswesen, ZUM 2019, 21, abrufbar unter https://osf.io/e5v7w/; Eisentraut (Fn. 14), S. 87; Schmidt (Fn. 12) passim; Krausnick (Fn. 16), S. 367; Bäuerle (Fn. 8), S. 1, Steinhauer (Fn. 41) passim.

52 Eine Liste findet sich bei Eisentraut/Hamann (Fn. 24), Abschnitt Open Access als Praxis der Rechtswissenschaft.

53 Erfolgreiche Open-Access-Zeitschriften stellt der von Hamann/ Hürlimann herausgegebene Tagungsband „Open Access in der Rechtswissenschaft" (Fn. 51) vor.

54 Erreichbar unter https://www.juwiss.de/.

55 Erreichbar unter https://verfassungsblog.de/.

56 Erreichbar unter https://voelkerrechtsblog.org/; dazu Kunz/ Riegner/Schmalz, Diskurse öffnen, Grenzen überwinden: Der Völkerrechtsblog als Plattform für globale Wissenschaftskommunikation im digitalen Zeitalter, in: Hamann/Hürlimann (Fn. 51).

57 In den Naturwissenschaften spielt der Preprintserver arXiv.org eine bedeutende Rolle; zum Aufschwung der Preprint-Server Schmeja, Beitrag auf dem TIB-Blog v. 27.3.2017, abrufbar unter https://blogs.tib.eu/wp/tib/2017/03/27/der-aufschwung-derpreprint-server/. 
etabliert und setzen daher ein proaktives Verhalten voraus. Dieser Kulturwandel vollzieht sich nur langsam. ${ }^{58}$ Denn die kommerzielle Veröffentlichung in etablierten Verlagen und Zeitschriften bildet auch heute noch einen wesentlichen Faktor für die Reputation von Rechtswissenschaftler ${ }^{*}$ innen. ${ }^{59}$ Obwohl es für die fachliche Qualität einer Veröffentlichung natürlich nicht darauf ankommt, wo sie veröffentlicht wird, spielt für die Reputation auch der Publikationsort eine zentrale Bedeutung. Denn nicht allein die wissenschaftliche Qualität einer Veröffentlichung entscheidet über ihren Erfolg; ganz wesentlich kommt es auch auf die soziale Akzeptanz im wissenschaftlichen Diskurs an. ${ }^{60}$ Hierbei erfüllen die schon etablierten und traditionell im Closed Access publizierenden Verlage und Zeitschriften eine „Gatekeeper“- oder auch Filterfunktion: ${ }^{61}$ Wer dort veröffentlicht, wird von der "scientific community“ wahrund ernst genommen. Zugespitzt formuliert: „Journale machen Karrieren!"62 Der insbesondere in den Naturwissenschaften sogar mathematisch berechnete sog. Impact Faktor einer Zeitschrift wirkt in der Rechtswissenschaft auch ganz ohne Zahlenunterbau: ${ }^{63}$ Während für Studierende noch im Wesentlichen zählt, welches Lehrbuch einen Inhalt am besten erklären kann, sind Rechtswissenschaftler*innen schnell in der Lage, eine rein an Reputation ausgerichtete "Rangliste“ der angesehensten Verlage und Zeitschriften zu erstellen. ${ }^{64}$ Verlage funktionieren insoweit wie Marken: Der Markenname allein reicht bereits, damit sich eine Veröffentlichung unter der Vielzahl konkurrierender Publikationen absetzt, mehr Aufmerksamkeit erhält und zu einem Reputationsgewinn führt.

\section{bb) Ökonomische Erwägungen}

Ein weiterer zentraler Einwand gegen Open Access und OER in der Rechtswissenschaft ist die ökonomische Rationalität der Wissenschaftler*innen: Für Aufsatzpublikationen gibt es häufig ein Honorar, während die Pub-

58 „Umdenkprozesse“ hält auch für erforderlich Gurlit (Fn. 39), S. 343 (353); skeptisch gegenüber einem grundlegenden Wandel Bäuerle (Fn. 8), S. 1 (7); einen flächendeckenden Systemwechsel hält erst langfristig für möglich Peukert (Fn. 14), S. 5; vom „Henne-Ei-Problem“ spricht Sosnitza, Google Book Search, Creative Commons und Open Access - Neue Formen der Wissensvermittlung in der digitalen Welt, RW 2010, S. 225 (238).

59 Haug (Fn. 14), S. 89 (90); der Begriff Reputation dient insofern als „Chiffre für die fachliche Qualität eines Wissenschaftlers“, Schulze-Fielitz, Staatsrechtslehre als Mikrokosmos, 2013, S. 188; Bäuerle (Fn. 8), S. 1 (7).

60 Schulze-Fielitz (Fn. 59), S. 369 f.; von Pfad- und Strukturabhängigkeiten spricht Peukert (Fn. 14), S. 4; zur Bedeutung des Publikationsortes auch Lutz, Zugang zu wissenschaftlichen Informationen in der digitalen Welt, 2012, S. $174 \mathrm{f}$.

$61 \operatorname{Haug}($ Fn. 14), S. 89 (90); vgl. auch Müßig, „Ein Knauf als Tür“: likation in Open-Access-Zeitschriften entweder kostenlos erfolgt oder von den Autor*innen sogar zusätzliche Mittel für die Veröffentlichung eingeworben werden müssen. Zur guten Finanzausstattung der traditionellen Verlage tragen dabei nicht nur die Bibliotheken bei, sondern auch die Rechtspraxis, also Anwaltschaft und Gerichte. Es ist kein Grund ersichtlich, warum die Rechtspraxis nicht weiterhin zugunsten der Wissenschaftler*innen an der Finanzierung der Forschung beteiligt werden sollte. ${ }^{65}$ Open Access auf dem goldenen Weg wird sich deshalb nur dann durchsetzen können, wenn gemeinsam mit der Rechtspraxis neue Formen der Finanzierung von Open-Access-Publikationen gefunden werden, bei der auch die Autor*innen für ihre Arbeit finanziell entlohnt werden. ${ }^{66}$

Auch für die Produktion von Ausbildungsliteratur werden die Wissenschaftlerinnen an den Verlagsgewinnen beteiligt. Auch hier besteht insofern ein monetärer Anreiz zum Verharren in hergebrachten Strukturen, soweit keine neuen Anreize geschaffen werden, auf eine Kommerzialisierung zulasten der Studierenden zu verzichten.

\section{Lösungsansätze}

Wie also könnte auf diese Vorbehalte reagiert und die Rechtswissenschaft zur digitalen Öffnung bewegt werden?

1. Zwang durch Open-Access-Zweitverwertungspflichten

Um dem Übertritt in das neue Zeitalter einer öffentlichen Wissenschaft den nötigen Spinn zu geben, kam man in Baden-Württemberg auf die Idee, die Hochschulen qua Änderung des Baden-Württembergischen Hochschulgesetzes nach $\$ 44$ Abs. 6 BWLHG dazu anzuhalten, ihr wissenschaftliches Personal zu Open-AccessZweitverwertungen in Anlehnung an die

Open-Access-Verpflichtung durch Forschungsförderung vs. Gemeinfreiheitsgrenzen digitaler Wissenschaftskommunikation, JZ 2015, S. 221 (227).

62 Ziegler, Die Bedeutung der Verlage wandelt sich, Forschung\&Lehre v. 8.6.2019, abrufbar unter https://www.forschung-und-lehre.de/zeitfragen/die-bedeutungder-verlage-wandelt-sich-1841/.

63 Näher zur Relevanz von Metriken für juristische Karrieren $\mathrm{Ha}$ mann/Hürlimann, Open Access - Was soll das?, in: dies. (Fn. 51), S. 3 (11 ff.).

64 Schulze-Fielitz (Fn. 59), S. 192.

65 Diesen Punkt betont auch Rux (Fn. 18), S. 70 (71).

66 Über die bisher bestehenden Geschäftsmodelle für Open-AccessPublikationen informiert https://open-access.net/informationenzu-open-access/geschaeftsmodelle/. 
Schrankenregelung des $\$ 38$ Abs. 4 UrhG und damit zum grünen Weg zu verpflichten. ${ }^{67}$ Das Hochschulgesetz spricht selbst von Zweitveröffentlichung, was jedoch ungenau ist, weil das urheberrechtlich geschützte Recht zur Veröffentlichung nur die Erstveröffentlichung erfasst; weitergehende Verwendungen des einmal publizierten Inhalts stellen urheberrechtlich nur noch Verwertungen der Erstpublikation dar, sodass im Folgenden von Zweitverwertung die Rede sein soll. ${ }^{68}$

Der Aufforderung des Landesgesetzgebers ist die Universität Konstanz mit einer Satzungsregelung nachgekommen. ${ }^{69}$ Danach werden die Voraussetzungen erfüllende Publikationen nach Ablauf der Jahresfrist auf dem universitätseigenen Repositorium hochgeladen sowie kostenlos und öffentlich zugänglich der Allgemeinheit zur Verfügung gestellt. Gegen die Regelung haben mehrere Professoren der Universität Konstan $z^{70}$ einen Normenkontrollantrag vor dem VGH Mannheim gestellt, der vom VGH nunmehr dem BVerfG vorgelegt wurde. ${ }^{71}$

Die Verpflichtung, wissenschaftliche Ergebnisse zugänglich zu machen, ist zwar keine gänzlich neue Idee des Gesetzgebers: Die Hochschulgesetze der Länder sehen schon jetzt verfassungsrechtlich unbedenkliche $\mathrm{Pu}$ blikationspflichten vor, insbesondere für Drittmittelforschung, aber auch darüber hinaus. ${ }^{72}$

Besondere Aufmerksamkeit erfährt die Baden-Württemberger Regelung jedoch deshalb, weil die Zweitverwertung Open Access erfolgen muss. Den Wissenschaftler*innen wird also einerseits das Recht genommen, über die Zweitverwertung autonom zu ent- scheiden; andererseits wird zugleich eine Entscheidung über die Form der Verwertung getroffen, wonach der Inhalt jedem offen zur Verfügung gestellt werden muss. Der so als Zwang formulierte Anspruch der Politik an eine freie Verbreitung von steuerfinanzierten wissenschaftlichen Erkenntnissen gerät in Konflikt mit zentralen grundrechtlichen Verbürgungen und auch mit dem Europäischen Unionsrecht.

\section{a) Zuständigkeit der Landesgesetzgeber}

Eine erste Achillesferse landesrechtlicher Zweitverwertungspflichten ist die grundgesetzliche Kompetenzordnung. Dies deshalb, weil der Schwerpunkt der Regelung ${ }^{73}$ vom VGH im Urheberrecht gesehen wird, das nach den Art. 71, 73 Abs. 1 Nr. 9 GG der ausschließlichen Bundeskompetenz zugewiesen ist. ${ }^{74}$ Wohl nicht zu bezweifeln ist, dass mit einer Zweitverwertungspflicht überhaupt das Urheberrecht berührt wird, weil die Regelung die sich an die Urheberschaft anschließenden Verwertungsrechte der $\$ \$ 15 \mathrm{ff}$. UrhG betrifft. ${ }^{75}$

Die Norm ist jedoch im Schwerpunkt - wie auch vom Landesgesetzgeber angenommen ${ }^{76}-$ dem in der Landeskompetenz liegenden Recht des Hochschulwesens zuzuordnen. Denn sie betrifft schwerpunktmäßig die Verbreitung der von dem wissenschaftlichen Personal der Hochschulen veröffentlichten Werke. Es geht bei der Regelung zwar auch um das Verwertungsrecht der Wissenschaftler*innen, im Schwerpunkt jedoch um eine Ausgestaltung der Forschungspraxis, ${ }^{77}$ wenn mit dem Gesetz der Zugang zu wissenschaftlichen Informationen erleichtert werden soll. Zugleich werden Pflichten der
67 Näher zum Hintergrund Hartmann, Zwang zum Open Access-Publizieren? Der rechtliche Präzedenzfall ist schon da!, LIBREAS. Library Ideas, 32 (2017), S. 1, abrufbar unter https:// edoc.hu-berlin.de/bitstream/handle/18452/19845/hartmann. pdf?sequence=1\&isAllowed=y; s. auch Höpfner/Amschewitz, Die Zweitveröffentlichungspflicht im Spannungsfeld von OpenAccess-Kultur und Urheberrecht, NJW 2019, S. 2966 (2966).

68 Darauf hinweisend Schulze (Fn. 12), §38 Rn. 25.

69 Satzung zur Ausübung des wissenschaftlichen Zweitveröffentlichungsrechts gem. $\$ 38$ IV UrhG, Satzung Nr. 90/2015; abrufbar unter https://www.uni-konstanz.de/typo3temp/secure_downlo ads/60959/0/4dba8009a4125316f7fa4c33872edd6f0ff01669/SatzungZweitveroeffentlichungsrecht.pdf.

70 Der offene Brief von Theile im Namen des Professoriums des Fachbereichs Rechtswissenschaft der Universität Konstanz ist abgedruckt in Löwisch, Konstanzer Juristenfakultät verweigert sich der Pflicht zur Zweitveröffentlichung, OdW 2016, 135, abrufbar unter http://ordnungderwissenschaft.de/wp-content/ uploads/2019/11/14_loewisch_konstanzer-juristenfakultaet_verweigert_zweitveroeffentlichungspflicht_odw_2016.pdf.

71 VGH Mannheim, Beschluß v. 26.9.2017, Az.: 9 S 2056/16, abrufbar unter http://lrbw.juris.de/cgi-bin/laender_rechtsprechung/document.py?Gericht=bw\&nr=22843 = VBlBW 2018, $166=$ ZUM 2018, 211.
72 S. Hartmer/Detmer, Hochschulrecht, 3. Aufl. 2017, 4. Kapitel Rn. 165; Bäuerle (Fn. 8), S. 1 (16); Steinhauer (Fn. 41), S. 57 f.; differenzierend hingegen Schmidt (Fn. 12), S. 81 ff., die die Pflichtenregelung im Berliner Hochschulgesetz für unverhältnismäßig hält.

73 Zur Schwerpunkttheorie des BVerfG vgl. BVerfG, Urt. v. 17.2.1998, Az.: 1 BvF 1/91 = BVerfGE 97, 228, 251 ff., abrufbar unter http://www.servat.unibe.ch/dfr/bv097228.html.

74 VGH Mannheim (Fn. 71), Rn. 77 ff.; ebenso Haug (Fn. 14), S. 89 (92); Schmidt (Fn. 12), S. 246 f.; Krausnick (Fn. 16), S. 367 (375 und 378).

75 VGH Mannheim (Fn. 71), Rn. 81; dies bezweifelt jedoch die Antragsgegnerin, a.a.O. Rn. 44; Hintergrund dieser Rechtsauffassung ist die im Urheberrecht vertretene Differenzierung zwischen Form und Inhalt bei der Gewährung von Urheberrechtsschutz, näher Schmidt (Fn. 12), S. 163 ff. sowie Götting/Lauber-Rönsberg (Fn. 15), S. 137 (141).

76 LT-Drs. 15/4684, S. 215 f., abrufbar unter https://www. landtag-bw.de/files/live/sites/LTBW/files/dokumente/WP15/ Drucksachen/4000/15_4684_D.pdf.

77 Zur Zuweisung der Ausgestaltung von Forschung und Lehre zur Landeskompetenz Uhle, in: Maunz/Dürig, GG, Art. 70 Rn. 103 (Lfg. 53 Oktober 2008). 
Hochschulmitglieder statuiert, wofür allein das Hochschulrecht der Länder berufen ist. ${ }^{78}$

b) Vereinbarkeit mit Art 5 Abs. 3 S. 1 GG

In materieller Hinsicht geraten Open-Access-Zweitverwertungspflichten in eine Spannungslage mit Art. 5 Abs. 3S. 1 GG.

\section{aa) Schutzbereich}

Die Wissenschaftsfreiheit schützt auch die sog. „Publikationsfreiheit", also die Freiheit nach eigener Beurteilung der Veröffentlichungsreife, des Veröffentlichungsorgans und des Veröffentlichungszeitpunktes darüber zu entscheiden, wissenschaftliche Erkenntnisse der Öffentlichkeit zugänglich zu machen. ${ }^{79}$ Davon umfasst ist die Entscheidung über das „wie“ und „wann“, über das „wo" sowie über das „ob“ der Veröffentlichung. ${ }^{80}$ In ihrer Ausgestaltung als Abwehrrecht umfasst die Publikationsfreiheit zudem das Recht, nicht zu publizieren. ${ }^{81}$

\section{bb) Eingriff}

Weder Open-Access-Erstveröffentlichungs-, noch Zweitverwertungspflichten greifen in diese negative Publikationsfreiheit ein. ${ }^{82}$ Denn die Open-Access-Verpflichtung trifft keine Aussage über eine generelle Pflicht zur Veröffentlichung, sagt also nicht, „ob“ veröffentlicht werden muss, sondern knüpft nur an das „wie“ der Veröffentlichung die Bedingung, dass diese Open Access zu erfolgen habe. ${ }^{8_{3}}$

Auch auf das „wie“ der Erstveröffentlichung wirkt sich die Zweitverwertungspflicht nicht aus. Eine mit der Pflichtenregelung begründete verlagsseitige Ablehnung eines Publikationswunsches wäre in Hinblick auf $\$ 38$ Abs. 4 UrhG unzulässig. ${ }^{84}$ Aufgrund dieses Zweitverwertungsrechts müssen alle Verlage eine potentielle Zweitverwertung bereits in ihre Kalkulation einbeziehen, sodass durch die baden-württembergische Regelung auch nicht die Gefahr besteht, dass den von der Pflicht betroffenen Wissenschaftler*innen schlechtere finanzielle Konditionen für die Publikation geboten werden. ${ }^{85}$

Ein Eingriff kann jedoch deshalb bejaht werden, weil sich eine Zweitverwertungspflicht auf die Publikationsrezeption auswirken kann. ${ }^{86}$ Eine Open-Access-Zweitverwertungspflicht berührt zudem das „wo" der Publikationsfreiheit, weil in das Recht der Wissenschaftler*innen eingegriffen wird, über den Ort der Zweitverwertung frei zu disponieren, wenn ein Upload des Beitrags auf dem universitären Repositorium erfolgt. ${ }^{87}$

\section{cc) Rechtfertigung}

Damit ist die Frage nach den Möglichkeiten einer Rechtfertigung von Open-Access-Zweitverwertungspflichten aufgeworfen. Die Wissenschaftsfreiheit steht nur unter dem Vorbehalt verfassungsimmanenter Schranken. ${ }^{88} \mathrm{Als}$ eingriffslegitimierendes Parlamentsgesetz steht $\$ 44$ Abs. 6 LHG BW Pate, ${ }^{89}$ in dem jedoch auch verfassungsimmanente Wertungen zum Ausdruck kommen müssten. Nicht ausreichend wäre es daher, wenn mit der Norm bloß gesellschaftspolitische Ziele verfolgt würden..$^{90}$ Der Idee der freien Verfügbarkeit von Forschungsliteratur müsste vielmehr verfassungsrechtlicher Rang zukommen, um eine Verpflichtung der Wissenschaft rechtfertigen zu können. ${ }^{91}$
78 S. das Vorbringen der Antragsgegnerin im Verfahren vor dem VGH Mannheim (Fn. 71), Rn. 42 ff.; Peukert (Fn. 14), S. 7 f.; i.E. ebenso Steinhauer (Fn. 41), S. $34 \mathrm{f}$.

79 Gärditz (Fn. 40), Art. 5 Abs. 3 Rn. 111.

80 Vgl. Gärditz (Fn. 40), Rn. 111 ff.; Schmidt (Fn. 12), S. 50; Fehling, Verfassungskonforme Ausgestaltung von DFG-Förderbedingungen zur Open-Access-Publikation, OdW 2014, S. 179 (191), abrufbar unter http://ordnungderwissenschaft.de/wp-content/ uploads/2019/11/24_fehling_dfg_odw_ordnung_der_wissenschaft_2014.pdf; anders aber Pflüger/Ertmann, E-Publishing und Open Access - Konsequenzen für das Urheberrecht im Hochschulbereich, ZUM 2004, S. 436 (441), die nur Publikationspflichten an wissenschaftsinadäquaten Veröffentlichungsorten als schutzbereichseröffnend ansehen; anders auch Peukert (Fn. 14), S. $19 \mathrm{ff}$.

81 Fehling (Fn. 80), S. 179 (191); Gärditz (Fn. 40), Rn. 103 sowie Rn. 113; auch dies ist nicht unbestritten geblieben, den Streitstand stellt dar Schmidt (Fn. 12), S. 50 ff.; s. zur a.A. Bäuerle (Fn. 7), S. 1 (14) sowie Pernice, in: Dreier, GG, 2. Aufl. 2004, Art. 5 III Rn. 28.

82 Peukert (Fn. 14), S. 19; so wohl auch Fehling (Fn. 80), S. 179 (212).

83 A.A., aber ohne nähere Begründung Krausnick (Fn. 16), S. 367
(378); undeutlich Haug (Fn. 14), S. 89 (92 f.).

84 Diese Gefahr sehen aber Haug (Fn. 14), S. 89 (93); Krausnick (Fn. 16), S. 367 (379); Steinhauer (Fn. 41), S. 60.

85 Fehling (Fn. 80), S. 179 (191).

86 Fehling (Fn. 80), S. 179 (191).

87 Vgl. Steinhauer (Fn. 41), S. 59.

88 Gärditz (Fn. 40), Rn. 151; Fehling (Fn. 80), S. 179 (197).

89 Zum Erfordernis Gärditz (Fn. 40), Rn. 152.

90 Fehling (Fn. 80), S. 179 (197).

91 Hier nicht vertieft, weil bisher nicht realisiert, wird die Rechtfertigung einer Verpflichtung zur Open-Access-Bereitstellung von Lehrmaterialien; hierfür kommt Art. 12 Abs. 1 GG als legitimierendes Grundrecht der Studierenden in Betracht, vgl. Gärditz (Fn. 40), Rn. 162; BVerfG, Beschl. v. 17.2.2016, Az.: 1 BvL 8/10 = BVerfGE 141, 143, Rn. 58, abrufbar unter http://www.servat. unibe.ch/dfr/bv141143.html ; s. zur Verfassungsmäßigkeit der Verpflichtung zu digitalen Lehrveranstaltungen in Zeiten von Corona auch Dorf/Hartmer, Ist elektronische Lehre Dienstpflicht?, Forschung\&Lehre v. 3.4.2020, abrufbar unter https:// www.forschung-und-lehre.de/recht/ist-elektronische-lehredienstpflicht-2667/. 
(1) Art. 5 Abs. 3 GG in seiner objektiv-rechtlichen Dimension

Dafür in Betracht kommt Art. 5 Abs. 3 GG selbst in seiner objektiv-rechtlichen Dimension. ${ }^{92}$ In dieser Dimension schützt die Wissenschaftsfreiheit die Funktionsfähigkeit und Effizienz der Wissenschaft und kann dafür auch Freiheitsbeeinträchtigungen gegenüber den Wissenschaftler*innen legitimieren. ${ }^{93}$

Ausprägung der Schutzfunktion ist auch die Förderung der Verbreitung wissenschaftlicher Erkenntnisse. ${ }^{94}$ Nicht erforderlich ist es dafür jedoch, dass ohne Zweitverwertungspflicht erhebliche Funktions- und EffizienzeinbuBen drohten und die wissenschaftliche Kommunikation über Forschungsergebnisse einzubrechen droht. ${ }^{95}$ Auch für die Rechtfertigung von Evaluationspflichten ist nicht etwa erforderlich, dass Lehre nahezu nicht mehr ordnungsgemäß stattfinde, sondern „nur“, dass die Qualität der Lehre sichergestellt werden soll. ${ }^{96}$

Den freien Fluss forschungsrelevanter Informationen fördernde Maßnahmen können schon dann auf die objektiv-rechtliche Dimension der Wissenschaftsfreiheit gestützt werden,wenn eine Open-Access-Verpflichtung die Wissenschaftsfreiheit in Hinblick auf die Zugänglichkeit forschungsrelevanter Publikationen stärken kann. ${ }^{97}$ Der Umweg über eine kommerzielle Verlagsveröffentlichung führt sowohl zu zeitlichen und ökonomischen Restriktionen bei der Publikation als auch bei der Rezeption, gerade auch in Anbetracht der im juristischen Publikationswesen zu beobachtenden Preissteigerungen..$^{98}$ Maßnahmen zugunsten einer freien Zugäng- lichkeit von Forschungsliteratur können somit auf die objektiv-rechtliche Dimension der Wissenschaftsfreiheit gestützt werden. ${ }^{99}$

(2) Art. 5 Abs. 3 GG als Grundrecht anderer

Wissenschaftler*innen

Auch das aus der Wissenschaftsfreiheit der anderen Wissenschaftler*innen fließende Recht auf Zugang zu Forschungsergebnissen kann als rechtfertigende Verfassungswertung herangezogen werden. ${ }^{100}$

(3) Informationsfreiheit

Fraglich ist hingegen, inwieweit die aus Art. 5 Abs. 1 S. 1 GG fließende Informationsfreiheit zugunsten von Open-Access-Verpflichtungen effektiviert werden kann. Zwar wird dem Grundrecht überwiegend kein Anspruch auf Zugänglichmachung von Informationen entnommen. ${ }^{101}$ Den Gesetzgeber trifft aber jedenfalls dann eine Schutzpflicht, wenn es zu Strukturund Konzentrationseffekten auf dem Medienmarkt kommt, die Informationsmonopole befürchten lassen..$^{102}$ Ob ein solcher die Schutzpflicht auslösender Status für die Rechtswissenschaft indes erreicht ist, ist zu bezweifeln. Die Verlagslandschaft kann als (noch) hinreichend diversifiziert gelten ${ }^{103}$ Mithin lässt sich die Informationsfreiheit (vorerst) nicht heranziehen, um Open-AccessVerpflichtungen zu rechtfertigen.

\section{(4) Staatliche Finanzierung}

Fraglich ist auch, ob die staatliche Finanzierung der Forschung als Gegenspieler der Wissenschaftsfreiheit her-
92 Gärditz(Fn. 40), Rn. 154; Fehling (Fn. 80), S. 179 (197); zurückhaltend hingegen Krausnick (Fn. 16), S. 367 (374); ebenso Sandberger, Die Zukunft wissenschaftlichen Publizierens, Open Access und Wissenschaftsschranke, Anmerkungen zu den Kontroversen über die Weiterentwicklung des Urheberrechts, OdW 2017, S. 75 (80) brufbar unter http://ordnungderwissenschaft.de/wp-content/ uploads/2019/11/11_2017_02_sandberger_die-zukunft-deswissenschaftlichen-publizierens_odw.pdf.

93 Haug (Fn. 14), S. 89 (94); Gärditz (Fn. 40), Rn. 154.

94 Haug (Fn. 14), S. 89 (93).

95 So aber Haug (Fn. 14), S. 89 (94); im Einzelnen ist umstritten, ab wann die objektiv-rechtliche Dimension der Wissenschaftsfreiheit Eingriffe in das Abwehrrecht legitimieren kann; differenzierend Fehling (Fn. 80), S. 179 (197).

96 Vgl. BVerfG, Beschl. v. 17.2.2016, Az.: 1 BvL 8/10 = BVerfGE 141, 143 Rn. 58, abrufbar unter http://www.servat.unibe.ch/ dfr/bv141143.html; Gärditz (Fn. 40), Rn. 155; zur grundsätzlichen Zulässigkeit von Evaluationspflichten auch zuletzt VGH BW, Urt. v. 19.12.2019, Az.: 9 S 838/18, abrufbar unter http:// lrbw.juris.de/cgi-bin/laender_rechtsprechung/document. py?Gericht $=$ bw\&nr $=30323$.

97 Dies jedenfalls für Förderbedingungen bejahend Fehling (Fn. 80), S. 179 (198), a.A. Haug (Fn. 14), S. 89 (93).
98 Hamann/Hürlimann (Fn. 63), S. 3 (28); Bäuerle (Fn. 8), S. 1 (11).

99 Bäuerle (Fn. 8), S. 1 (10 f.); Vgl. auch Peukert (Fn. 14), S. 20.

100 Steinhauer (Fn. 41), S. 71 f. spricht hier von „Recherchefreiheit“; Dieses Interesse referenziert auch Schulze (Fn. 12), $\$ 48$ Rn. 25; Peukert (Fn. 14), S. 21; Bäuerle (Fn. 8), S. 1 (14), der diese Problematik auf der Ebene des Schutzbereichs der Wissenschaftsfreiheit ansiedelt; a.A. Schmidt (Fn. 12), S. 93.

101 Grabenwarter, in: Maunz/Dürig, GG, Art. 5 Abs. 1, 2 Rn. 1021 (Stand: 85. Lfg. November 2018); Krausnick (Fn. 16), S. 367 (375); Fehling (Fn. 80), S. 179 (198).

102 Fehling (Fn. 80), S. 179 (198 f.); enger Grabenwarter (Fn. 101), Rn. 1022, der demokratiegefährdende Konzentrationstendenzen verlangt.

103 Vgl. Rux (Fn. 18), S. 70 (71); Die Anwendbarkeit der Informationsfreiheit dennoch befürwortend Fehling (Fn. 80), S. 179 (199); von einer Monopolstruktur gehen aus Hamann/Hürlimann (Fn. 63) S. 3 (22), die die jeweiligen Artikel bzw. die gesamte Fachzeitschrift als Monopolprodukt qualifizieren; indes sind die jeweiligen rechtlichen Inhalte gerade nicht unter dem Urheberrecht monopolisierbar, vgl. Schulze (Fn. 12), \$2 Rn. 93; dass die Bibliotheken Zeitschriften möglichst umfassend erwerben, liegt an ihrem Aufgabenzuschnitt. 
angezogen werden kann. ${ }^{104}$ Zwar verlangen die hergebrachten Grundsätze des Berufsbeamtentums nach Art. 33 Abs. 5 GG auch Geltung gegenüber verbeamteten Hochschullehrer*innen und kommen als Rechtfertigungsgrund in Betracht. ${ }^{105}$ Dies gilt jedoch nur in Hinblick auf nichtwissenschaftliche Dienstaufgaben, die äußere Form der Diensterfüllung und die Einhaltung allgemeiner Regeln, während der Zugriff auf Wissenschaftsinhalte verwehrt ist. ${ }^{106}$ Eine Verfügungsbefugnis des Staates über die Forschungsergebnisse der Wissenschaftler*innen ist daher abzulehnen; ${ }^{107}$ gleiches gilt für eine Anbietungspflicht gegenüber dem Dienstherrn von in der Dienstzeit entstandenen Werken. ${ }^{108}$

\section{(5) Abwägung}

Die kollidierenden Verfassungsgüter (also die Wissenschaftsfreiheit im Dreiecksverhältnis Autor*in - andere Wissenschaftler*innen - objektiv-rechtliche Dimension) sind im Wege praktischer Konkordanz in Ausgleich zu bringen. ${ }^{109}$

Fraglich ist dabei insbesondere, ob Open-AccessZweitverwertungspflichten zum Schutz der Funktionsfähigkeit der Wissenschaft erforderlich und angemessen sind.

So wird bezweifelt, ob eine Open-Access-Zweiverwertungspflicht überhaupt nötig sei, da sich der Status quo in den Rechtswissenschaften deutlich von der Situation in anderen Fachbereichen unterscheide: Während etwa in den Naturwissenschaften der Zugang zu Forschungsliteratur aufgrund von Monopolstrukturen als mitunter prekär qualifiziert werden könne, ${ }^{110}$ sei in der Rechtswissenschaft bisher die Funktionsfähigkeit gewahrt. ${ }^{111}$ Dieser These ist jedoch begründet entgegen getreten worden: Eine Erhebung unter 35 rechtswissenschaftlichen Fachzeitschriften hat ergeben, dass auch in der deutschen Rechtswissenschaft erhebliche und schnelle Steigerungen der Zeitschriftenpreise erfolgt sind. ${ }^{112}$ Dies hat auch Auswirkungen auf die Bibliotheksetats, weshalb Bibliotheken durchaus in der Bereitstellung des Zugangs zu Fachliteratur eingeschränkt sind. ${ }^{113}$

In Hinblick auf die Angemessenheit ist zu berücksichtigen, dass es sich bei der Pflicht nur um eine Zweitverwertungspflicht handelt. Der Eingriff in die Wissenschaftsfreiheit wiegt also weniger schwer, als wenn eine Open-Access-Erstveröffentlichungspflicht normiert worden wäre. Für ein nur sehr leichtes Gewicht des Eingriffs spricht zudem, dass von der Zweitverwertungspflicht betroffene Veröffentlichungen zuvor in ClosedAccess-Zeitschriften publiziert wurden; hierbei werden in der Regel sämtliche Verwertungsrechte an den Verlag abgetreten. Die von der Zweitverwertungspflicht betroffenen Wissenschaftler*innen hätten also auch ohne die Pflicht gar nicht das Recht, außerhalb der Regelung des $\$ 38$ Abs. 4 UrhG über die Zweitverwertung zu disponieren.

Andererseits muss eine Open-Access-Zweitverwertungspflicht aber den berechtigten Interessen der Wissenschaftler*innen Rechnung tragen und darf etwa dann nicht statuiert werden, wenn erstveröffentlichte Erkenntnisse überholt sind oder sich als falsch herausgestellt haben, die Erstpublikation aufgrund gesetzlicher Vorschriften zurückgezogen worden ist oder die Publikation Rechte Dritter verletzt; hierauf nimmt $\$ 4$ Abs. 1 der Konstanzer Satzung auch Rücksicht. $\$ 44$ Abs. 6 S. 2 LHG BW schafft die Grundlage, um den in der Wissenschaft Tätigen die von Art. 5 Abs. 3 S. 1 GG geforderte Mitwirkung bei der Ausgestaltung einer entsprechenden Regelung im Rahmen des Erlasses einer Hochschulsatzung zu ermöglichen. ${ }^{114}$ Auch wurden mittlerweile mit den Repositorien der Hochschulen wissenschaftsadäquate Open-Access-Publikationsinfrastrukturen - jedenfalls für Open-Access-Zweitveröffentlichungen - geschaffen. ${ }^{115}$ Damit stellt sich die Open-AccessZweitverwertungspflicht - jedenfalls in der Ausgestaltung
104 Dass die Finanzierung durch Personal- und Sachmittel der öffentlichen Hand erfolgt, ist ein regelmäßig zugunsten von Open Access vorgetragenes Argument, s. nur Bäuerle (Fn. 8), S. 1 (5).

105 Gärditz (Fn. 40), Rn. 176.

106 Gärditz (Fn. 40), Rn. 176.

107 Haug (Fn. 14), S. 89 (93).

108 Höpfner/Amschewitz (Fn. 67), S. 2966 (2970); Schmidt (Fn. 12), 1. Teil Kapitel 2 und 3 sowie 2. Teil Kapitel 1 passim; Steinhauer (Fn. 41), S. 26 ff.; vertiefend auch Lutz (Fn. 60), S. 207 ff.

109 Zur praktischen Konkordanz allgemein Schladebach, Praktische Konkordanz als verfassungsrechtliches Kollisionsprinzip - Eine Verteidigung, Der Staat 2014, 263; für Art. 5 Abs. 3 GG s. Pernice, in: Dreier, GG, 2. Aufl. 2004, Art.5 III Rn. 42; Schübler-Pfister, in: Gärditz/Pahlow (Hrsg.), Hochschulerfinderrecht, 2011, Teil 1 Rn. 22.
110 Zahlen finden sich bei Pflüger/Ertmann (Fn. 80), S. 436 (437).

111 In diese Richtung für die Rechtswissenschaft Gärditz (Fn. 40), Rn. 158 Fn. 9; ebenso Haug (Fn. 14), S. 89 (94).

112 Hamann/Hürlimann (Fn. 63) S. 3 (22 ff.).

113 Hamann/Hürlimann (Fn. 63) S. 3 (29).

$114 \mathrm{Zu}$ den Mitwirkungsrechten vgl. zuletzt BVerfG, Beschl. v. 5.2.2020, Az.: 1 BvR 1586/14 Rn. 16, abrufbar unter https://www. bundesverfassungsgericht.de/e/rk20200205_1bvr158614.html.

115 Diese Forderung, jedoch in Hinblick auf eine Open-Access-Erstpublikationspflicht, formuliert Peukert (Fn. 14), S. 23 ff.; als Fachrepositorium in der Rechtswissenschaft fungiert $<\operatorname{intR}>{ }^{2} \operatorname{Dok}[\S]$, erreichbar unter https://intr2dok.vifa-recht.de/content/index.xml; zum Repositorium Mathieu, Open Access für die Rechtswissenschaft: Pflicht oder Privatsache? Eine bibliothekarische Perspektive, in: Hamann/Hürlimann (Fn. 51), S. 203 (205 f.). 
durch die Universität Konstanz im Zusammenspiel mit dem baden-württembergischen Gesetzgeber - als gerechtfertigter Eingriff in die Wissenschaftsfreiheit dar. ${ }^{116}$

c) Vereinbarkeit mit Art. 14 Abs. 1 und 12 Abs. 1 GG

Während die Produktion und Publikation von Forschungs- und Lehrmaterialien der Wissenschaftsfreiheit unterfällt, wird deren wirtschaftliche Verwertung überwiegend den Schutzbereichen der Eigentums- und Berufsfreiheit zugeordnet. ${ }^{117}$

Der Urheber genießt den Schutz des Art. 14 Abs. 1 GG. ${ }^{118}$ Als Inhalts- und Schrankenbestimmung bedarf die Open-Access-Zweitverwertungspflicht der Rechtfertigung, dies erst Recht, als das Gesetz keinen ausgleichenden Vergütungsanspruch des Urhebers vorsieht. ${ }^{119}$ Beschränkungen des Verwertungsrechts können dabei nur durch ein gesteigertes öffentliches Interesse gerechtfertigt werden, ${ }^{120}$ das jedoch in Anbetracht des Interesses an bestmöglicher Wissenschaftskommunikation anzunehmen ist. ${ }^{121}$ Die Regelung stellt sich auch als verhältnismäßig dar, da bei der Erstveröffentlichung typischerweise bereits über die weitergehenden Verwertungsrechte abschließend disponiert wurde. ${ }^{122}$

Auch in Hinblick auf die Berufsfreiheit lässt sich ein Eingriff im Sinne einer Berufsausübungsregelung aufgrund der Reduktion der Erwerbschancen zwar annehmen. Dieser ist in Hinblick auf die zugleich ermöglichte erleichterte Wissenschaftskommunikation jedoch ebenfalls als gerechtfertigt anzusehen. ${ }^{123}$ d) Vereinbarkeit mit der Urheberrechtsrichtlinie

Schließlich sind Zweifel an der Vereinbarkeit von OpenAccess-Zweitverwertungspflichten mit der europäischen Urheberrechtsrichtlinie $^{124}$ angemeldet worden. ${ }^{125}$ Dies deshalb, weil die Urheberrechtsrichtlinie in Art. 5 einen abschließenden ${ }^{126}$ Schrankenkatalog für Eingriffe in das ausschließliche Recht des Urhebers zur öffentlichen Zugänglichmachung vorsieht. Bezweifelt worden ist, ob Open-Access-Zweitverwertungspflichten von diesem Schrankenkatalog erfasst werden können. ${ }^{127}$ Nach Art. 5 Abs. 3 lit. a der Richtlinie können die Mitgliedstaaten Ausnahmen oder Beschränkungen für Zwecke der wissenschaftlichen Forschung zulassen, sofern die Quelle, einschließlich des Namens des Urhebers angegeben wird und soweit dies zur Verfolgung nicht kommerzieller Zwecke gerechtfertigt ist. Genau hierum handelt es sich im Falle der Zweitverwertungspflicht, wenn bereits publizierte Artikel auf universitätseigenen Repositorien online gestellt werden. ${ }^{128}$ Auch den weiterhin nach Art. 5 Abs. 5 der Richtlinie erforderlichen „Dreistufentest" besteht die Open-Access-Zweitverwertungspflicht, weil sie legitime Zwecke verfolgt, weder die normale Verwertung des Werkes beeinträchtigt, noch die berechtigten Interessen des Rechtsinhabers ungebührlich beeinträchtigt werden. $\$ 38$ Abs. 4 UrhG sieht ja bereits jetzt schon ein Zweitveröffentlichungsrecht vor, sodass verlagsseitige Verwertungsrechte nicht tangiert sind. ${ }^{129}$ Auch eine ungebührliche Beeinträchtigung der
116 Anders stellte sich die Bewertung einer Open-Access-Erstveröffentlichungspflicht dar, weil hierdurch den Wissenschaftler*innen weitreichend auch die Möglichkeit zur freien Disposition über den Ort der Erstveröffentlichung genommen wäre.

117 Höpfner/Amschewitz (Fn. 67), S. 2966 (2969); Bäuerle (Fn. 8), S. 1 (10); differenzierend hingegen Gärditz (Fn. 40), Rn. 90 ff.

118 Haug (Fn. 14), S. 89 (94); Dreier, in: Dreier/Schulze, Urheberrechtsgesetz, 6. Aufl. 2018, Einleitung Rn. 39; nicht jedoch die Verlage, vgl. Haug (Fn. 14), S. 89 (94 f.); ebenso Höpfner/Amschewitz (Fn. 67), S. 2966 (2970); Fehling (Fn. 80), S. 179 (186); Peukert (Fn. 14), S. 16 f.

119 Ein solcher wurde vom BVerfG in der Entscheidung zum Schulbuchprivileg für erforderlich erachtet, s. BVerfG, Beschluß v. 7.7.1971, Az.: 1 BvR 765/66 = BVerfGE 31, 229, abrufbar unter http://www.servat.unibe.ch/dfr/bv031229.html.

120 Dreier (Fn. 118), Rn. 39.

121 Haug (Fn. 14), S. 89 (94); a.A. aber Höpfner/Amschewitz (Fn. 67), S. 2966 (2970), die eine Vergütungsregelung fordern.

122 I.E. ebenso Krausnick (Fn. 16), S. 367 (374).

123 Haug (Fn. 14), S. 89 (95); Höpfner/Amschewitz (Fn. 67), S. 2966 (2971); Krausnick (Fn. 16), S. 367 (375); anders läge die Sache hingegen im Falle einer Open-Access-Erstveröffentlichungspflicht, weil eine solche den Urhebern die Kommerzialisierung der Publikation gänzlich unmöglich machen würde; a.A. aber Peukert (Fn. 14), S. 30; erforderlich wäre dann zumindest eine Vergütungsregelung wie etwa in $\$ 42 \mathrm{Nr}$. 4 ArbnErfG.

124 RL 2001/29/EG des Europäischen Parlaments und des Rates vom 22.5.2001 zur Harmonisierung bestimmter Aspekte des Urheberrechts und der verwandten Schutzrechte der Informationsgesellschaft, abrufbar unter https://eur-lex.europa.eu/LexUriServ/ LexUriServ.do?uri=OJ:L:2001:167:0010:0019:DE:PDF.

125 Haug (Fn. 14), S. 89 (91 f.); im Ergebnis auch, aber differenzierender Höpfner/Amschewitz (Fn. 67), S. 2966 (2971 ff.); zu den grundfreiheitlichen Implikationen näher Krausnick (Fn. 16), S. 367 (371 ff.).

126 S. Erwägungsgrund 32 der Richtlinie.

127 Es wurde schon in Hinblick auf $\$ 38$ Abs. 4 UrhG diskutiert, ob es sich hierbei um einen Verstoß gegen die Schrankenregelung handele; dies lehnen richtigerweise ab Höpfner/Amschewitz (Fn. 67), S. 2966 (2972).

128 Zweifel in Hinblick auf die Ausschließlichkeit der wissenschaftlichen Zweckrichtung verfängt nicht, so überzeugend Höpfner/ Amschewitz (Fn. 67), S. 2966 (2972).

129 Anders, dabei aber diesen Aspekt unberücksichtigt lassend Höpfner/Amschewitz (Fn. 67), S. 2966 (2972). 
Interessen des Rechtsinhabers ist nicht festzustellen, insofern kann auf die Erwägungen zur Angemessenheit der Grundrechtsbeeinträchtigung verwiesen werden. ${ }^{130}$ Ein Verstoß gegen die Urheberrechtsrichtlinie scheidet damit ebenfalls aus.

\section{Förderung}

Auch wenn Open-Access-Zweitverwertungspflichten demnach aus verfassungs- und unionsrechtlicher Sicht als zulässig zu bewerten sind, sollte die Öffnung der Rechtswissenschaft keine erzwungene sein, sondern auf die freie Entscheidung der Wissenschaftler*innen zurückgeführt werden. ${ }^{131}$ Um einen Kulturwandel zu erreichen, sollte die Politik dafür das Anreizinstrumentarium effektivieren und die Wissenschaftsförderung gezielter als bisher dazu nutzen, um ihr Interesse an einer Öffnung der Rechtswissenschaft Gewicht zu verleihen. Forschungsförderung an die Bedingung einer Open-Access-Publikation der Forschungsergebnisse und an das Forschungsprojekt begleitende Wissenschaftskommunikation zu knüpfen, ${ }^{132}$ wird auf weitaus größere Einsicht der Rechtswissenschaftler*innen stoßen und kommt auch weitgehend ohne Konfliktlage zu grundrechtlichen Verbürgungen aus. ${ }^{133}$
Dafür reichen die bisherigen Bemühungen jedoch nicht. Mittlerweile haben die Hochschulen zwar von der DFG geförderte ${ }^{134}$ Open Access-Förderfonds aufgesetzt, aus denen nicht nur Publikationsgebühren für OpenAccess-Zeitschriftenartikel, sondern teilweise auch die Kosten für Open-Access-Monographien erstattet werden können. ${ }^{135}$ Indes widersprechen die Förderbedingungen den in der Rechtswissenschaft etablierten Fachkulturen und finden daher nur wenig Interesse: So fließen die Fördergelder vollständig den Verlagen zu, während die Wissenschaftler*innen leer ausgehen. Auch eine Förderung von Open-Access-Veröffentlichungen in etablierten Closed-Access-Zeitschriften wird häufig ausgeschlossen, was der Reputationskultur in der Rechtswissenschaft zuwiderläuft. ${ }^{136}$

Auch die Drittmittelforschung wird bisher nicht vollständig von Open-Access-Veröffentlichungspflichten abhängig gemacht. ${ }^{137}$ Die DFG etwa fordert dies zwar, zwingt aber nicht dazu. ${ }^{138}$ Öffentliche Fördermittel für OER-Projekte sind zudem rar. ${ }^{139}$

\section{Freiwilligkeit: $\$ 38$ Abs. 4 UrhG}

Wen abseits von Kommerzialisierungsinteressen die Idee einer offenen Wissenschaft überzeugt und wer
130 Anders wiederum Höpfner/Amschewitz (Fn. 67), S. 2966 (2972 f.), die die Zahlung einer angemessenen Vergütung an den Urheber für erforderlich erachten.

131 So auch Steinhauer (Fn. 41), S. 48.

132 Zum Modell des Wissenschaftsförderungsrechts in den USA und dem Vereinigten Königreich Peukert (Fn. 14), S. 5 f.; Förderung ebenfalls befürwortend Sandberger (Fn. 92), S. 75 (80); weitere Anreizinstrumente finden sich bei Schmidt (Fn. 12), S. 249: Mittelvergabe orientiert an Open-Access-Veröffentlichungen; Open-Access-Veröffentlichungen als Gegenstand von Berufungsvereinbarungen.

133 Eine grundrechtliche Bindung privater Forschungsförderer scheidet von vorneherein aus; eine Spannungslage zu den grundrechtlichen Verbürgungen der Wissenschaftler*innen kann nur dort entstehen, wo die Grundausstattung betroffen ist; ansonsten handelt es sich bei der Forschungsförderung nämlich schon um keinen Grundrechtseingriff, sondern um eine freiheitserweiternde Leistung, vgl. umfassend zu dieser Frage Fehling (Fn. 80), S. 179; Schmidt (Fn. 12), S. 119 ff. sowie 249 f.; vgl. auch Gärditz (Fn. 40), Rn. 104 sowie Steinhauer (Fn. 41), S. 73 ff.

134 Förderprogramm „Open Access Publizieren“, https://www.dfg.de/ foerderung/programme/infrastruktur/lis/lis_foerderangebote/ open_access/.
135 So ko-finanziert bspw. die Freie Universität Berlin nunmehr in einer Pilotphase auch Publikationskosten für Open-AccessMonographien und -Sammelbände; nähere Informationen unter https://www.fu-berlin.de/sites/open_access/finanzierung/ monogr-sammelbaende/index.html.

136 Auch wenn die Kritik am „double dipping“ bei sog. hybriden Open-Access-Modellen durchaus berechtigt sein mag, vgl. hierzu näher Mittermaier, Double Dipping beim Hybrid Open Access Chimäre oder Realität?, Informationspraxis Bd. 1, Nr. 1 (2015), 1, abrufbar unter http://dx.doi.org/10.11588/ip.2015.1.18274.

137 Zwangsmodelle sind bereits auf europäischer Ebene im Rahmen des Förderprogramms Horizon 2020 (s. bereits Fn. 49 sowie https://www.horizont2020.de/einstieg-open-access.htm) realisiert; auch am „Plan S“ beteiligte nationale Förderorganisationen wollen Forschungsförderung von Open-Access-Verpflichtungen abhängig machen.

138 DFG-Vordruck 2.00 - 12/15, S. 44, abrufbar unter https://www. dfg.de/formulare/2_00/v/dfg_2_00_de_v1215.pdf.

139 Wikimedia Deutschland e.V. (Hrsg.) (Fn. 23), S. 60; einen ersten Beitrag leisten Förderprojekte wie das Fellow-Programm Freies Wissen; Finanzierungsformen wie Crowd-Sourcing könnten eine Alternative darstellen, vgl. Deimann/Neumann/Muuß-Merholz (Fn. 19), S. 35. 
einen Beitrag zur Idee global verfügbaren, frei zugänglichen Wissens beisteuern will, kann auch heute schon ganz einfach seinen Beitrag leisten.

Aufsatzpublikationen werden sich künftig auch reputationsfördernd in Open-Access-Zeitschriften unterbringen lassen. ${ }^{10}$ Wer bisher in Closed-Access-Journalen veröffentlicht und davon erst einmal nicht absehen möchte, kann bis dahin seine Aufsätze auf dem grünen Weg der Allgemeinheit zur Verfügung stellen. Zeitschriftenaufsätze dürfen im Rahmen der Regelung des $\$ 38$ Abs. 4 UrhG $^{141}$ nach einer Embargofrist von einem Jahr Open Access zugänglich gemacht werden. Die Erfahrungen des Autors dieses Beitrags zeigen, dass die Zeitschriften häufig damit einverstanden sind, dafür auch die gesetzte Manuskriptversion zur Verfügung zu stellen. Die Datei lässt sich einfach auf einem universitätseigenen Repositorium hochladen und ist dann - auf dem sog. grünen Weg - Open Access verfügbar.

Dissertationen und andere Monographien müssen nicht im Verlag, sondern können auch auf den universitären Repositorien kostenlos Open Access veröffentlicht werden. Für alle, die auf eine Verlagsveröffentlichung aus Reputationsgründen nicht verzichten können, bieten immer mehr Verlage auch Open-Access-Modelle an. Die damit häufig verbundenen höheren Kosten werden durch eine umfassende Sichtbarkeit der eigenen Forschung ausgeglichen. ${ }^{142}$

In Hinblick auf Lehrmaterialien stellen alle mit Lehraufgaben Betrauten auch heute schon sehr viel Material kostenlos zur Verfügung. Über eine offene Lizenzierung und einen Upload außerhalb der geschlossenen Lernplattformen der Universitäten könnten diese einem weit größeren Nutzerkreis zur Verfügung gestellt werden.

\section{Fazit}

Die Digitalisierung öffnet einen Weg, in der all unser Wissen frei im digitalen Raum verfügbar sein könnte. $\mathrm{Ob}$ wir diesen Weg gehen oder Wissen weiter hinter Bezahlschranken verstecken wollen, liegt in der Hand eines jeden von uns. Nehmen wir also mit Kant den Ausgang aus der selbstverschuldeten Unmündigkeit ${ }^{143}$ und entscheiden künftig bewusst darüber, wie wir Forschungsergebnisse und Lehrmaterialien veröffentlichen möchten. Dass in der Rechtswissenschaft bisher wenig von den Möglichkeiten öffentlicher Forschung und Leh-

140 Hamann/Hürlimann (Fn. 63), S. 3 (13).

141 Vor Erlass der Norm wurde intensiv um die Zulässigkeit dieser als Recht ausgestalteten Regelung gerungen; s. dazu Krausnick (Fn. 16), S. 367 (376 ff.); Fehling (Fn. 80), S. 179 (183 f.); Bäuerle (Fn. 8), S. 1 (16); Sandberger, Zweitverwertungsrecht, ZUM 2013, 466; die Regelung für unions- und verfassungswidrig erachtend hingegen Müßig (Fn. 61), S. 221 (229 ff.); Sprang, Zweitveröffentlichungsrecht - ein Plädoyer gegen $₫ 38$ Abs. 4 UrhG-E, ZUM re Gebrauch gemacht wird, hat Gründe, die nicht durch Zwangssysteme überspielt, sondern im konstruktiven Dialog mit der Politik beseitigt werden sollten. Gleichzeitig sollten wir uns nicht hinter vorgeschobenen Gründen verstecken, sondern bewusst mit den Potentialen einer öffentlichen Rechtswissenschaft auseinandersetzen und von den schon heute bestehenden Möglichkeiten mutiger Gebrauch machen.

\footnotetext{
Der Autor ist Wissenschaftlicher Mitarbeiter am Fachbereich Rechtswissenschaft der Freien Universität Berlin sowie Rechtsreferendar am Kammergericht Berlin. Er ist Herausgeber des ersten offen lizenzierten Lehrbuchs zum Verwaltungsrecht "Verwaltungsrecht in der Klausur" sowie des Open-Access-Fallrepetitoriums „Fälle zum Verwaltungsrecht".
}

2013, 461

142 S. Steinhauer, Zur Sichtbarkeit und Verbreitung rechtswissenschaftlicher Dissertationen, in: Hamann/Hürlimann (Fn. 51), S. 31.

143 Kant, Beantwortung der Frage: Was ist Aufklärung, Berlinische Monatsschrift, Dezember 1784, S. 481, abrufbar unter http:// www.deutschestextarchiv.de/book/show/kant_aufklaerung_1784. 